\title{
Classical conditioning of an "avoidance" response in goldfish using a linear presentation procedure
}

\author{
SUMIO IMADA, KEIJI HAGIMOTO, and HIROSHI IMADA \\ Kwansei Gakuin University, Nishinomiya, Japan
}

\begin{abstract}
The present study examined the nature of the "avoidance" response in goldfish under the linear presentation procedure (Zerbolio, 1981). With this procedure, shuttling behavior occurring during the presentation of the trial stimulus produces either CS- or CSt, and further occurrence of shuttling within the trial interval $(10 \mathrm{sec})$ changes the value of CS from negative to positive, or vice versa. If the fish remains in the compartment when the prevailing cue state is CS- at the end of the interval, shock can be avoided. With this procedure fish responded to the CS+ more than to the CS- and avoided shock. But fish in one of two control groups, in which responses had no effect in changing the cue state from CS+ to CS-, or vice versa, also showed a clear differentiation. The results were generally in line with the view that the "avoidance" response in fish is acquired through classical conditioning. The contribution of classical conditioning to the acquisition of avoidance response is discussed.
\end{abstract}

One of the major problems regarding shuttlebox avoidance learning in goldfish is whether they learn to avoid the signaled shock by way of a classical or an instrumental conditioning process (Zerbolio, 1980). Woodard and Bitterman $(1971,1973)$ found that their instrumentally trained goldfish did not respond during the CS interval significantly more than did classically trained subjects: "It seems then that all of our data on avoidance conditioning in goldfish can be accounted for without reference to a process of instrumental learning"' (Woodard \& Bitterman, 1973, p. 128). In contrast to this conclusion, however, Zerbolio and Wickstra (1978a, 1978b) concluded that avoidance acquisition in goldfish occurred through an instrumental process. In light of the paucity of evidence in this regard (cf. Scobie \& Fallon, 1974), however, it may be premature to conclude that the shuttlebox avoidance response is learned by an instrumental conditioning process.

Recently, Zerbolio and Wickstra $(1979 \mathrm{~b}, 1980)$ introduced a Y-maze, consisting of three identical alleys, which they used with a new avoidance procedure-a simultaneous presentation procedure. With this procedure, a fish was required to choose between the CS - and CS + alleys, ${ }^{1}$ starting from the alley in which it stayed when the trial started. They assumed that if the fish increased responding to the "CS-," this could be taken as evidence for instrumental conditioning.

In order to apply this simultaneous presentation

This research was supported by a Grant-in-Aid for Scientific Researches (No. 57510060), Ministry of Education of Japan, to Hiroshi Imada. The authors' mailing address is: Department of Psychology, Kwansei Gakuin University, Nishinomiya, 662, Japan. procedure to a two-way shuttlebox situation, Zerbolio (1981) devised a method he calls a "linear presentation procedure". In this procedure, shuttling behavior occurring during the presentation of the trial stimulus (TS) produces either CS- or CS + , and further occurrence of shuttling within the trial interval changes the value of the CS from negative to positive, or vice versa. If the fish remains in the compartment when the prevailing cue state is CS- at the end of the interval, shock can be avoided. Zerbolio found that his fish kept responding until they found themselves exposed to the CS-. On the basis of this finding, he concluded that avoidance acquisition in fish was through an instrumental process.

Although the fish's choice of CS- over both CS+ and TS seems convincing, one can still argue that the shuttling response in fish was elicited classically by the stimuli's being paired with shock (CS+ and TS), and that it was not elicited by the CS- because CShad never been paired with shock (cf. Woodard \& Bitterman, 1973). Zerbolio (1981) used a control group in which the light colors of the CS + and CSwere made the same so they became indistinguishable.

In the present experiment, Zerbolio's (1981) experiment using the linear presentation procedure was replicated, but different control groups were used. In one of the control groups, fish were trained under the linear presentation procedure by the classical conditioning method. Thus, the first response (R1) to TS produced either $\mathrm{CS}+$ or $\mathrm{CS}-$, but further responses did not change the cue state. If avoidance acquisition in fish occurs through an instrumental process, there is no reason to make further response to $\mathrm{CS}+$, and thus differentiation in responding to $\mathrm{CS}+$ and $\mathrm{CS}-$ in this control group should not develop. 


\section{METHOD}

\section{Subjects}

Thirty 5- to 7-cm-long naive goldfish (Carassius auratus) served as subjects. They were housed in a tank and fed regular dry food after their daily training session. The water was aerated and filtered, and its temperature was approximately $25.5^{\circ} \mathrm{C}$.

\section{Apparatus}

A shuttle tank, similar to the one described by Horner, Longo, and Bitterman (1961), was used. The inside dimensions of the tank were $26.3 \mathrm{~cm}$ (length), $11.5 \mathrm{~cm}$ (width), and $10.3 \mathrm{~cm}$ (height). Each compartment was separated by a 6.3-cm center hurdle with 60-deg sloping sides and a 11.3-cm-long flat top. Water clearance over the hurdle was maintained at $2 \mathrm{~cm}$. Shuttling activity was monitored by photoswitches (OMRON, OPE-S100) across the top of the hurdle at the ends of the top flat portion, $6.2 \mathrm{~cm}$ apart. Photobeams were invisible.

The trial stimulus (TS) was the onset of an overhead yellow lamp $(7 \mathrm{~W}, 100 \mathrm{~V} \mathrm{ac})$, located $50 \mathrm{~cm}$ above the apparatus. The intensity of this light was $41.3 \mathrm{~lx}$ when measured on the hurdle surface, directing the illuminometer to the light source. It provided reasonably uniform illumination to the entire tank. Constant illumination was provided by a lamp $(5 \mathrm{~W}, 100 \mathrm{~V}) 100 \mathrm{~cm}$ above the apparatus with an intensity of $4.24 \mathrm{~lx}$. The conditioned stimuli were provided by red or green lamps lit simultaneously at both ends of the shuttle tank. The intensities of these lights were 14.5 and $9.4 \mathrm{~lx}$, for the red and green lights, respectively, when measured on the hurdle-top surface, directing the illuminometer to the light sources. For half of the subjects of each group, $\mathrm{CS}+$ was the red lamp and CS- was the green lamp, and for the other half, this relationship was reversed. Shock was delivered via $25.4 \times 10 \mathrm{~cm}$ stainless steel plates that lined the interior sides of the tank. The intensity and duration of the shock were $7.5 \mathrm{~V}$ ac $(0.65 \mathrm{~V} / \mathrm{cm})$ and 200 msec, respectively. Shocks were normally presented twice with an intershock interval (ISI) of $800 \mathrm{msec}$.

\section{Procednre}

During the first stage of the experiment (Days 1-3), only activities were monitored. The animals were then divided into three groups approximately matched in body size and the amount of activity observed during Days 1-3. On each day of the second stage of the experiment (Days 4-10), each group had 40 training trials (19 $\mathrm{CS}+$ trials and $21 \mathrm{CS}-$ trials), ${ }^{2}$ with a variable interval of $60 \mathrm{sec}$ (range: $40-100 \mathrm{sec}$ ) between trial of fet and the next trial onset. The trial started with the onset of the TS, and the goldfish's first response (R1), if it occurred at all, produced CS+ or CS-, in a predetermined random order.

For the instrumental conditioning group (Group 1), responses after $R 1$ could change the cue state from CS + to CS-, or vice versa. If $\mathbf{R l}$ produced $\mathrm{CS}+$, the fish, to avoid the shocks, had to make a second response (R2) within $10 \mathrm{sec}$ after the onset of the trial. If, however, the cue state after $R 1$ was $C S-$, the fish could avoid the shocks by making no further response. Any crossing between the start of the trial (T0) and the end of the trial (T11.2) changed the cue state. If the fish did not make $R 1$, it received double shocks, as described above, beginning $10 \mathrm{sec}$ after TS onset (T10). Then the TS went off after the second shock or at T11.2. The cue state always went back to the no-cue state of the intertrial interval after T11.2.

For the classical conditioning group (Group C), responses after R1 did not change the cue state, shocks were delivered after T10 on CS + trials, and no shocks were given on CS- trials. If the fish did not respond and the cue state remained TS, then the shocks were presented with a probability of $0.475(19 / 40)$, according to a predetermined order, to eliminate the avoidance contingency from $R 1$ to TS. In this group, the fish received shocks only on CS+ trials classically without regard to its responses.

In the control group (Group CC), the fish could change the cue state from TS to CS+ (or to CS-) by $R l$ in the same way as could the above two groups. But $R 2$ had no further effect, as with
Group C. But, unlike Group C, the specific cue state did not correspond to the shock and the fish received shocks classically in a predetermined order on 19 of 40 trials without regard to its responses or specific cue states.

\section{RESULTS}

The most important point in this study was whether the fish of Group $C$ responded differentially to $\mathrm{CS}+$ and $\mathrm{CS}-$. Figure 1 presents the median percentage of R2 to CS+ and CS- separately, which were produced by the fish's initial responses (R1s) to TS. For Groups I and C, the response differentiations are obvious. The Wilcoxon matched-pairs signed-ranks test (Siegel, 1956) conducted for the overall differences in responding to $\mathrm{CS}+$ and $\mathrm{CS}-$ confirmed these differentiations for Group I [T(10) $=0, \mathrm{p}<.01]$ and Group $\mathrm{C}[\mathrm{T}(10)=3, \mathrm{p}<.01]$. But for Group CC, for which there was no CS+ and $\mathrm{CS}-$, there was no difference in responding to the two different stimuli $[T(6)=5]$. The fish in Group C seemed to have responded more than those in Group I to both CS+ and CS-, but in neither case were the differences statistically significant by the Mann-Whitney $U$ tests (CS+ trials, $U=34.5 ; C S-$ trials, $U=30$ ).

For the fish in Group I, this differentiation was effective in avoiding shocks. The median percentages of avoidance in Group I were 5.0, 21.2, 22.5, 31.2, $38.7,40.0$, and 50.0 from Day 1 to Day 7 , respectively. On the other hand, the fish in Groups $C$ and $\mathrm{CC}$ received shocks without regard to their responses on 19 of the 40 daily trials.

Fish in Group I did not always display the same response patterns as those in Group C. Figure 2 shows the median percentages of R1 (left half) and R2 (right half) for the three groups. Responding to TS (R1) gradually increased over days in Groups I and C, but no such tendency was observed in Group CC. MannWhitney $U$ tests were conducted for Days 1-7 for all possible combinations of groups, and the only significant difference in responding to TS was between Groups I and $\mathrm{CC}(\mathrm{U}=7, \mathrm{p}<.002)$. Withingroups comparisons in the total number of responses between the first 2 days (Day 1-2) and the last 2 days (Day 6-7) revealed that Groups I and C made sig-

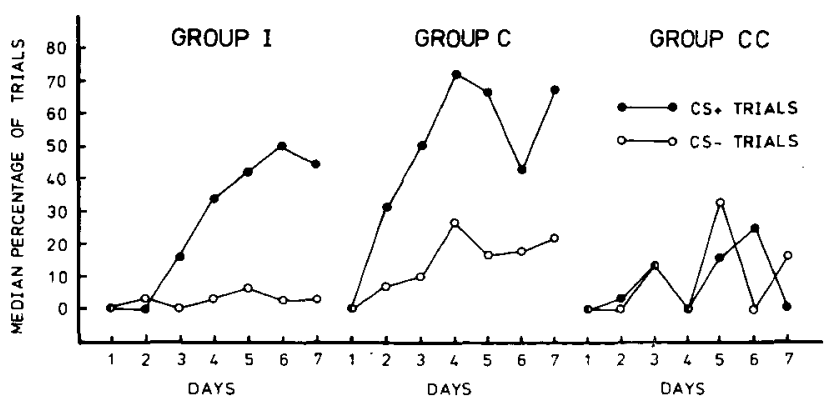

Figure 1. Median percentage of $\mathrm{R2}$ for $\mathrm{CS}+$ and $\mathrm{CS}-$ trials separately for Groups I, C, and CC. 


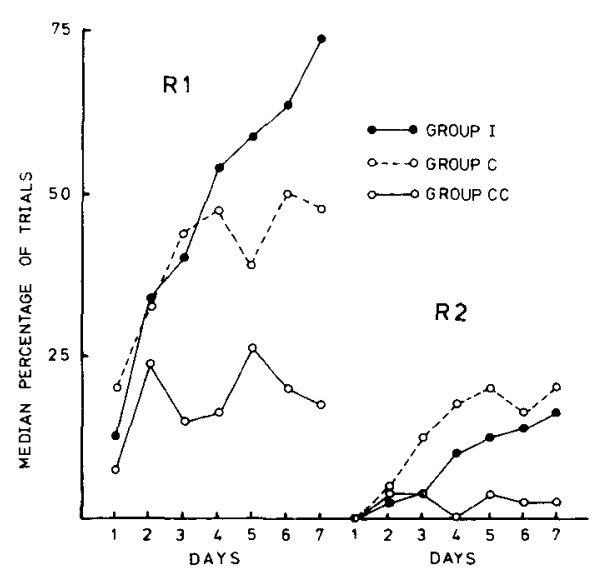

Figure 2. Median percentage of trials for fish in Groups I, C, and CC showed R1 and R2.

nificantly more $\mathbf{R} 1$ responses in the last 2 days than in the first 2 days [for Group I, $T(10)=0, p<.01$; for Group $C, T(10)=1, p<.01]$, but the difference was not significant for Group CC [T(9) $=10.5]$.

Although the opportunities for R2 were fewer for the fish in Group $C$ than for those in Group I, Group C shows more R2 than does Group I. Again, the results of the $\mathbf{U}$ tests, conducted for $\mathbf{R} 2$ on Days 17 for possible combinations of groups, showed a significant difference only between Groups $\mathrm{C}$ and $\mathrm{CC}$ $(\mathrm{U}=22, \mathrm{p}<.05)$. From these results, it is reasonable to conclude that fish in Group I had a tendency to emit more R1 than those in Group $\mathrm{C}$, but the tendency to respond further by making $R 2$ was less in Group I than in Group C.

These differences were partially anticipated from the latency data. The median latency of $R 1$ for Group I on Day 7 was $6.4 \mathrm{sec}$, but for Group C was $4.4 \mathrm{sec}(U=23, p<.05)$. Apart from the question of why the fish in Group C showed more rapid R1 than those in Group I, it is reasonable to consider that in Group $\mathbf{C}$ fish had more opportunities to make R2 (and rarely $\mathbf{R} 3$ ).
Table 1 shows the daily changes of median number of intertrial responses (ITRs) for each group (first rows). For easy comparisons with the changes of frequencies of responses during the 10-sec trial intervals (shown in the bottom rows), ITRs per $10 \mathrm{sec}$ are shown in the middle rows. The numbers in the bottom rows include $R 1, R 2$, and $R 3$, occurring to both $\mathrm{CS}+$ and $\mathrm{CS}-$, excluding unconditioned responses elicited by the shock. The ITRs do not differ significantly among groups, and they tend to decrease slightly over days, although not significantly. It may be noted that the responses occurring during the trial intervals were specifically elicited by the CSs in Groups I and C.

\section{DISCUSSION}

This experiment was performed to try to determine whether or not the "avoidance" response under the linear presentation procedure (Zerbolio, 1981; Zerbolio \& Royalty, 1982) could be interpreted as classsical conditioning. Based on the fact that the fish in Group $\mathrm{C}$ responded more to CS + than to CS-, as did Group I, it is possible to regard the avoidance response under the linear presentation procedure as classically conditioned. At least one should recognize the great contribution of classical conditioning to the acquisition of the avoidance response (e.g., Woodard \& Bitterman, 1971, 1973). Although Zerbolio (1981) emphasized the response-contingent stimulus change and its function of reinforcing the fish's "choice" of CS - alley, the present experiment seems to suggest that their "choice" responses may also be elicited by stimuli paired with shocks.

There is a question of whether the goldfish in our Groups I and C did actually discriminate CS+ and CS-. Asking such a question is important, inasmuch as Zerbolio and Wickstra (1979a) have recently demonstrated the possibility of explaining discriminated patterning in goldfish in terms of pseudoconditioning. The possibility of pseudoconditioning is

Table 1

Median Numbers of Intertrial Responses (First Row in Each Group), of the ITRs/10 sec (Second Row), and of the Response Frequencies During the Trial Intervals (Third Row) of Each Group

\begin{tabular}{|c|c|c|c|c|c|c|c|}
\hline & \multicolumn{7}{|c|}{ Days } \\
\hline & 1 & 2 & 3 & 4 & 5 & 6 & 7 \\
\hline \multicolumn{8}{|c|}{ Group I } \\
\hline \multicolumn{8}{|c|}{ Group C } \\
\hline CRs/10 sec & 0.20 & 0.35 & 0.57 & 0.65 & 0.60 & 0.66 & 0.66 \\
\hline \multicolumn{8}{|c|}{ Group CC } \\
\hline ITRs & 18 & 11 & 12 & 8 & 11 & 5 & 4 \\
\hline ITRs $/ 10 \mathrm{sec}$ & 0.08 & 0.05 & 0.05 & 0.03 & 0.04 & 0.02 & 0.01 \\
\hline CRs/10 sec & 0.07 & 0.23 & 0.18 & 0.17 & 0.30 & 0.20 & 0.21 \\
\hline
\end{tabular}


unlikely in our experiment for the following reasons: First, all shocks in the present experiment were signaled. The Zerbolio and Wickstra procedure of eliminating $\mathrm{CS}+$ and presenting the unsignaled shocks would have led to a greater amount of contextual conditioning and hence to pseudoconditioning. Second, in the present experiment, the ITIs were random within the range of $40-100 \mathrm{sec}$, which made temporal conditioning unlikely to occur. In other words, a pseudoconditioning-like phenomenon due to temporal conditioning was unlikely. Third, and most importantly, is the fact that there were very few ITRs in all groups, as shown in Table 1, which discounts the possibility of conditioning to contextual cues. On the basis of the above arguments and because of the differential occurrences of responses to CS+ and CS - in both Groups I and C, one can conclude that the classical conditioning contingency makes an important contribution in the present experiment.

An important problem with regard to classical differential conditioning is whether the response differentiation shown in Figure 1 by Group $C$ was due to the conditioning of excitation to CS + , to the conditioning of inhibition to CS-, or to both. In order to assess these possibilities, the results of Group CC shown in Figure 1 provide a good control, because, for this group, CSs were paired with shocks $50 \%$ of the time and neither excitation or inhibition should have been conditioned to the CSs. Comparing the Group CC curves with the CS- curve for Group C, it seems apparent that the response differentiation observed in Group $\mathbf{C}$ was due to conditioning of excitation to CS +. There is no tendency for the CS- curve of Group $C$ to be suppressed below the level of the curves for Group CC.

Opinions may vary with regard to what makes the control group proper in an experiment of the present kind. One may suggest a control group yoked with an avoidance group, thus eliminating the avoidance contingency and matching the number of shocks received in the two groups (Wickstra \& Zerbolio, 1979). What was important in the present experiment, however, was the fact that the differential conditioning procedure was used and that discrimination did occur in both Group I and Group C. The difference in the number of shocks received by these groups is not important. In a sense, the fact that discrimination was observed in Group C but not in Group CC was sufficient for the present purpose; the Group I data may be regarded as only supplementary.

Thus, the contribution of the classical contingency in the "avoidance" of goldfish cannot be denied. This does not necessarily mean that the "avoidance" in goldfish is acquired only through a classical process. Although not obtained systematically, our observational data suggest a difference in the way in which Groups I and C reacted to the TS: Upon presentation of the TS, Group I subjects hesitated, for a moment, to show smooth swimming, whereas Group $C$ subjects tended to react more immediately. Further experiments in which fish's behavior is systematically observed may clarify the nature of "avoidance" in goldfish.

\section{REFERENCES}

Horner, J. L., Longo, N., \& Bitterman, M. E. (1961). A shuttle box for fish and a control circuit of general applicability. American Journal of Psychology, 74, 114-120.

Scobie, S. R., \& Fallon, D. (1974). Operant and Pavlovian control of a defensive shuttle response in goldfish. (Carassius auratus). Journal of Comparative and Physiological Psychology, 86, 858-866.

Sieger, S. (1956). Nonparametric statistics: For the behavioral sciences (International student ed.). Tokyo: McGraw-Hill Kogakusha.

Wickstra, L. L., \& Zerbolio, D. J., JR. (1979). A voidance learning in goldfish: $A$ within-trials response-contingent successive discrimination paradigm. Paper presented at the annual meeting of the Midwestern Psychological Association, Chicago.

Woodard, W. T., \& Bitterman, M. E. (1971). Classical conditioning of goldfish in the shuttlebox. Behavior Research Methods \& Instrumentation, 3, 193-194.

Woodand, W. T., \& BitTerman, M. E. (1973). Pavlovian analysis of avoidance conditioning in the goldfish (Carassius auratus). Journal of Comparative and Physiological Psychology, 82, 123-129.

Zerbolio, D. J., JR. (1980). An application of the comparative method: Is conditioned avoidance a classical or instrumental process in goldfish? In M. R. Denny (Ed.), Comparative psychology (pp. 168-189). New York: Wiley.

Zerbolio, D. J., JR. (1981). Discriminated avoidance learning and reversal by goldfish in a shuttlebox using a linear presentation procedure. Animal Learning \& Behavior, 9, 346-356.

Zerbolio, D. J., JR., \& Royalty, J. L. (1982). Acquisition and extinction of differential responses to signals paired with shock or shock omission in goldfish: Evidence for truly discriminated avoidance learning. Animal Learning \& Behavior, 10, 377-389.

Zerbolio, D. J., J H., \& Wick8tha, L. L. (1978a). Does elimination of a negative phototaxis eliminate CAR acquisition in goldfish? Bulletin of Psychonomic Society, 11, 324-326.

Zerbolio, D. J., JR., \& Wickstra, L. L. (1978b). Goldfish avoidance acquisition: Is the process classical, instrumental, or a phototaxis? Bulletin of Psychonomic Society, 11, 321-323.

Zerbolio, D. J., Jn., \& Wickstra, L. L. (1979a). Discriminated response patterning in goldfish with successive presentation and explicitly unpaired pseudoconditioning procedures. Bulletin of the Psychonomic Society, 13, 307-310.

ZERBolio, D. J., JR., \& Wickstra, L. L. (1979b). Instrumentally based conditioned avoidance response acquisition in goldfish in a simultaneous presentation task. Bulletin of the Psychonomic Society, 13, 311-313.

Zerbolio, D. J., JR., \& Wickstra, L. L. (1980). Instrumental avoidance acquisition by goldfish in a $Y$-maze using explicit and response-contingent cues. Animal Learning \& Behavior, 8, 304-310.

\section{NOTES}

1. Zerbolio and Wickstra called the stimulus paired with an unconditioned stimulus "S-," but we adopt a more general name.

2. The number of CS+ and CS- trials should have been 20 each. This unequal number of CS + and $C S-$ trials was due to a mistake in programming, which was only realized later.

(Manuscript received July 7, 1983; revision accepted for publication February 10, 1984.) 